\title{
ARTIZT: Applying Ambient Intelligence to a Museum Guide Scenario
}

Oscar García, Ricardo S. Alonso, Fabio Guevara, David Sancho, Miguel Sánchez, and Javier Bajo

\begin{abstract}
Museum guides present a great opportunity where the Ambient Intelligence (AmI) paradigm can be successfully applied. Together with pervasive computing, context and location awareness are the AmI features that allow users to receive customized information in a transparent way. In this sense, Real-Time Locating Systems (RTLS) can improve context-awareness in AmI-based systems. This paper presents ARTIZT, an innovative AmI-based museum guide system where a novel RTLS based on the ZigBee protocol provides highly precise users' position information. Thus, it can be customized the content offered to the users without their explicit interaction, as well as the granularity level provided by the system.
\end{abstract}

Keywords: Ambient Intelligence, museum guide, context-aware, location-aware, Real Time Locating Systems, ZigBee.

Oscar García

School of Telecommunications, University of Valladolid, Paseo de Belén 15,

47011 Valladolid, Spain

e-mail: oscgar@tel.uva.es

Ricardo S. Alonso

Department of Computer Science and Automatic, University of Salamanca,

Plaza de la Merced, s/n, 37008, Salamanca, Spain

e-mail: ralorin@usal.es

Fabio Guevara · David Sancho

Nebusens, S.L. R\&D Department, Parque Científico de la USAL, Edificio M2,

Calle Adaja s/n, 37185, Villamayor, Salamanca, Spain

e-mail: \{fabio.guevara, david.sancho\} @nebusens.com

Miguel Sánchez · Javier Bajo

Pontifical University of Salamanca, C/ Compañía 5, 37002, Salamanca, Spain

e-mail: jbajope@upsa.es

P. Novais et al. (Eds.): Ambient Intelligence - Software and Applications, AISC 92, pp. 173-180. springerlink.com

(c) Springer-Verlag Berlin Heidelberg 2011 


\section{Introduction}

Ambient Intelligence (AmI) systems have to take into consideration the context in which they are used. That is, they must have context-awareness properties and adapt their behavior without the need of users to make an explicit decision or interact with them, allowing applications to be more usable and efficient [1]. People, places and objects are recognized as the three main entities when dealing with Ambient Intelligence [2]. The place where the user is and the objects that surround him determine the behavior of the system, thus obtaining in a natural way personalized, adaptive and immersive applications.

Mobile devices, such as smart phones, PDAs or tablets, offer a wide range of possibilities to create new AmI-based systems. One important feature of these devices is the ability to know their position, which includes the location of users themselves and any other object that is part of the environment [2]. However, the use of these devices on context-aware applications requires locating them more precisely. In this sense, Real-Time Locating Systems (RTLS) acquire a great importance in order to improve the applications based on the knowledge of the relative position of each user or object at any time.

One of the areas of interest where AmI becomes more relevant is Museum Guides applications [3]. Museum scenarios are environments where users receive a wealth of information from many sources. New information and communication technologies facilitate that the characteristics and related information about artworks can be offered in a more understandable, attractive and easy way to visitors. In this sense, the context information becomes relevant in order to personalize the experience for every user at every moment [4]. Thus, RTLS are presented as a resource that greatly improves context-awareness in AmI applications as these systems provide the position of every static or dynamic object that interacts throughout the scenario. There are different technologies that can be used when designing and deploying an RTLS, such as Global Positioning System (GPS) [5], Infrared (IR) Pointing Systems [6], Passive and Active Radio-Frequency Identification (RFID) [7], Wireless Local Area Networks (WLANs) [8] or Near Field Communication (NFC) [9].

There are several works that use RTLS to enhance the visitors experience in museums $[8,10]$. The ultimate goal of all these approaches is to find an association between the visitors and the art-work they are looking at a particular time. However, most of these solutions require a strong interaction between the visitors and the technology. That is, visitors must be close enough to the art-work to be detected, even bringing their devices to a particular object or waiting for other visitors to finish their interaction. Furthermore, these approaches do not provide enough accuracy to customize information with a high granularity level.

This paper describes ARTIZT, an innovative museum guide system in which visitors use tablets to receive personalized information and interact with the rest of the elements in the environment. An RTLS based on ZigBee is proposed in order to improve context-awareness. This system achieves a location precision of less than one meter. Thus, ARTIZT knows at every time how customized content must be shown to the visitors, based on the area on which they are located. This way, 
visitors can naturally walk throughout the museum receiving relevant information as they get closer to each art-work. With this approach, every art-work, zone or important detail of the museum can be contextualized.

The rest of the paper is structured as follows: Section 2 presents existing museum guide works, as well as the shortcomings that motivate the development of a new system. Then, Section 3 describes the basic components of ARTIZT, including the most important locating and context management technologies involved on it and how they are used. Finally, conclusions and future work are depicted.

\section{Problem Description and Related Work}

In recent years, ongoing advances in technology and communications have allowed people to be surrounded by mobile devices. These devices are increasingly powerful, easy-to-use and capable of communicating with each other in more innovative ways. These capabilities allow the creation of Ambient Intelligence systems: users' mobility is guaranteed and they are, day by day, more accustomed to the use of technology for any task.

A museum scenario is an ideal environment where AmI can improve the way the information is offered to visitors. AmI features encourage the creation of new museums guide approaches where information is presented in a natural, personalized and attractive way to users with a better human-machine interaction. This way, as art-works are usually statically placed in the environment, the main goal is how to detect where visitors are. If we can determine, as precisely as possible, the position of the visitor inside the museum, we will be able to know the entire context that surrounds him every time.

Multiple technologies can be used in order to determine the position of the visitors. The ultimate goal of knowing the positioning of the visitors is to provide information, as accurate as possible, about the art-works they are watching. In this sense, tagging the context and the use of RTLS are two widely used alternatives [11]. Next, it is analyzed the most used technologies in both trends.

When talking about RTLS, the first technology that comes into our mind is GPS. It has been used in context-aware tourist guides where users receive personalized information according to their position [12]. However, it only works outdoors and it is not an appropriate technology to develop an indoor museum guide. Some approaches try to solve indoor locating problem using combined technologies along GPS. Cyberguide [5] determines the users' position by means of infrared sensors. However, it is needed a direct line of sight between user and sensors, so it does not work properly in crowded environments. Exploring the use of infrared sensors, the HIPPIE system delivers information about the location of user in relation to an object [6]. Visitors point the art-work (which is provided with an infrared detector) and the system knows where they are and provides the content. Nevertheless, this solution requires a proactive user and, as mentioned before, direct line of sight and proximity between user and object. The action performed by the user when pointing the object (e.g., an art-work) is physically the same as is done in solutions that use RFID [7] or NFC technologies [9]. Both technologies 
follow the same pattern of performance: each object all over the museum is tagged. RFID or NFC tags, containing a unique identification number, are placed near the object. If visitors want to receive information about an art-work, they have to place their device nearby the tag. Then, the system identifies the object and loads the relevant information. The necessity of proximity between devices and tags makes these solutions non-transparent to the user as it requires a direct collaboration. Moreover, if multiple users want to see the information about the same art-work, they must do it one by one at each time. Bluetooth solves the peerto-peer relation between RFID tags and users [14]. The Eghemon system uses mobile phones that receive information via Bluetooth as users get closer to a piece of information (e.g., an art-work). These pieces can offer information to multiple devices simultaneously, but they must be close enough to them. If two pieces are close enough, the mobile device can only receive information from just one of them. Bluetooth can also be used to improve the devices locating. Bruns et al. have designed a solution where a grid of Bluetooth emitters is deployed throughout the museum, so the visitors' mobile phones transmit their position to the nearest emitter [10]. However, location is not as accurate as desirable, because the system only knows which mobile phones are associated to an emitter. Furthermore, Bluetooth can only support the association of up to seven mobile phones simultaneously to an emitter. The UbiCicero system [14] uses Active RFID to get the position of the users. In this case, users carry an RFID reader that continuously reads signals from active tags that are close to art-works. Active RFID technology allows a better locating approach because users do not have to be as close as with passive RFID technology to detect the object. However, location information is not precise enough because the system only detects which art-work is closer the users, so two objects that are close enough can cause interference and "confuse" the system. It is also possible to locate mobile devices that provide information on the museum via Wireless LANs. In this case, multiple Wireless LANs are created, usually one network for each different zone. When visitors change from a zone to another, devices automatically connect to an available network through which contents are provided [8]. This solution presents several problems: different coverage areas must not be overlapped; locating provides a poor precision (never gets precision under 2 meters); and infrastructure deployment is a hard process since it is needed a thorough calibration of all devices [13].

As can be seen along this section, there are many approaches that have been considered to create museum guides. Analyzing all of them, two ways of tackling the problem can be identified. The first one is the direct physical interaction between the visitor and the art-work. The second one consists of obtaining information without having a voluntary interaction by the visitor, where the system automatically provides information depending on visitors' location. ARTIZT follows the second approach. The main objective is to make interaction as transparent as possible to visitors. For doing that, it is very important that context information is precise enough. This way, an accurate location of visitors is the most important aspect of this approach. Therefore, it is easy to know which elements (i.e., art-work) surround each visitor, no matter several of these elements are separated by a short distance between them. Thus, it is possible to get a whole 
description of the context that surrounds each visitor in a more precise way. Based on the context information, the content provided to the visitors changes dynamically. Next section describes the basic features of ARTIZT and the way it works.

\section{ARTIZT: Ambient Intelligence Real-Time Locating System Museum GuIde Over Zigbee Technology}

The AmI paradigm proposes the development of applications that provide new ways of interaction between people and technology, adapting them to the needs of individuals and their environment [2]. ARTIZT (Ambient intelligence Real-Time locating system museum guIde over Zigbee Technology) follows these premises and offers personalized contents to the visitors in a transparent way according to the context information.

The potential of ARTIZT lies in the precision with which contextual information can be collected. In this sense, an innovative RTLS provides the system with users' positions with an error less than one meter, so it can be determined at any time which art-works are on the visitors' radio of interest, thus adapting precisely the information that it is provided to each user.

\subsection{The Real-Time Locating System}

The RTLS used is by ARTIZT is based on the novel n-Core platform [15], which is intended to develop ZigBee applications and provides both wireless physical devices and an Application Programming Interface (API) to access their functionalities. A network of ZigBee devices must be deployed all over the museum. This network is composed of a set of Sirius A (Figure 1 left) and Sirius Dongle devices (Figure 1 right). The Sirius A devices are placed across the ceiling of the museum (Figure 2), forming a network in which it is known the specific location of each one, as well as the relative positions with each of its neighbors (i.e., closest devices). The Sirius Dongle devices are inserted in tablet PCs that are carried by the visitors. This way, the visitors can move freely through the museum.
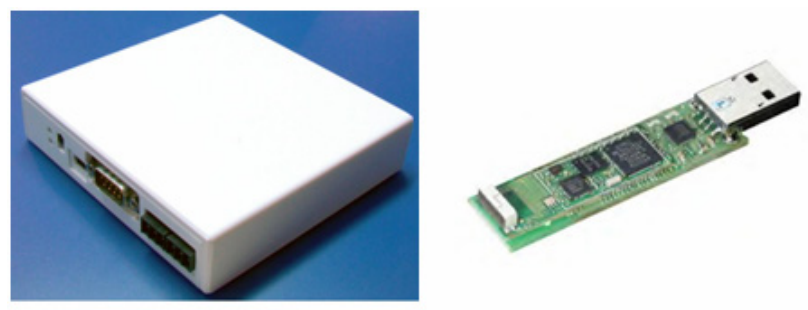

Fig. 1 Sirius A (left) and Sirius Dongle (right) devices

All devices communicate via the ZigBee standard. ZigBee is a low cost, low power consumption wireless communication standard, developed by the ZigBee Alliance. It is based on the IEEE 802.15.4 protocol and operates at the 
868/915MHz and $2.4 \mathrm{GHz}$ unlicensed bands. ZigBee is designed to be embedded in consumer electronics, home and building automation or toys and games. ZigBee allows star, tree or mesh topologies. Devices can be configured to act as network coordinator (creates and controls the network) router (sends/receives/forwards data to/from other devices) and end device (sends/receives data to/from other devices in the network).

Over this network infrastructure it is implemented a locating engine that provides users positioning whose accuracy reaches less than one meter. The infrastructure is completely dynamic and scalable so new devices can be added at any time without affecting the rest of the network. The operation of the RTLS is very simple. Visitors carrying mobile devices (i.e., tablets) move freely around the museum. The mobile devices send periodically a broadcast signal by means of the Sirius Dongle connected to them. The signal is received by the Sirius A devices placed all over the museum. The location engine, allocated in a central server, calculates the positions of all Sirius Dongle devices and therefore the position of every visitor. Once the system knows the location of each visitor, an application installed on the devices of the visitors customizes the information which is provided dynamically.

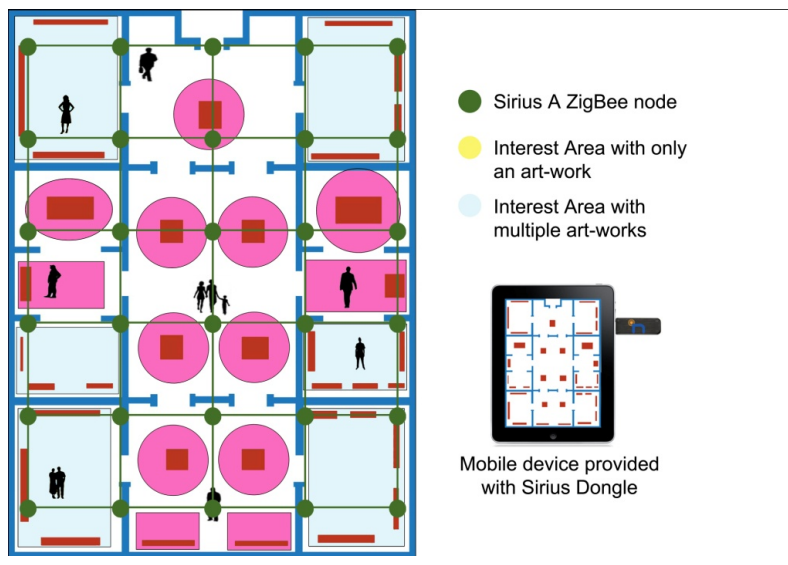

Fig. 2 Sirius A wireless network, Interest Areas and mobile device provided with Sirius Dongle

\subsection{Context Information Management}

Once ARTIZT gets the position of the visitors, the information must be personalized according to every visitor status. Each user carries a tablet on which it is installed a light and user-friendly application developed specifically for the museum. Contextual information of the museum is included in this application so that, from the position of the visitors, ARTIZT decides which information is shown in the device. To do this it is created a map of the museum and, with the 
location information received by the server, it is determined the location of visitors continuously. In addition, each level of the museum is divided into "Interest Areas" (see Figure 2). So when a user enters into one of these, the application customizes all the information that is wanted to be shown to visitors. Reader can realize that with a so precise RTLS, these areas can be as small as desired, so that the system can provide enhanced context information and personalize it always in a transparent way without any user interaction.

Tablets' application contains all the information that may be provided to users. Thus, ZigBee network only carries data from the devices to the server, necessary to calculate their position, and the location of the visitors from the server to the tablets. These communications reduce traffic data and there are always alternative paths in the network so fault tolerance increases and data loss is minimized.

ARTIZT is configured to provide the customized content. When the position of the visitor is known, and using a series of data that is collected before the visitor starts his route, the system is able to tailor the information that is being shown in the form of content or language.

\section{Conclusions and Future Work}

Transparency and customized data is one of the keys in the development of AmI applications. If these applications want to adapt their behavior depending on the context that surrounds users transparently, it is important that users' location is as precise as possible.

Museum guides are a scenario where applying AmI techniques becomes more meaningful: multiple contextual information that contains the museum and the great mobility of the visitors make their location and the data filtering process become challenges to solve in an efficient way.

ARTIZT is a museum guide system that includes AmI techniques. This system makes use of a powerful RTLS to determine where visitors are. This RTLS, based on an innovative ZigBee-based platform, provides high precision in the locating process. Therefore, the information that surrounds each visitor at any moment can be precisely determined and provided to them on real-time. The use of the ZigBee network to transport locate information minimize data loss (visitors' devices contains all the necessary information) and reduce the risk of disconnection, as there are always alternative ways to reach the server over the ZigBee grid.

Future work on ARTIZT includes the use of sensors in order to determine any information that may affect the context (temperature, pressure, humidity, etc.) and estimate the direction of art-works and visitors through the use of compasses. Combining the precise location information provided by the RTLS with the information from compasses it will be possible to determine where visitors are looking at, enhancing the user experience.

Acknowledgments. This work has been supported by the Spanish Ministry of Science and Innovation, Project T-Sensitive, TRA2009_0096. 


\section{References}

1. Baldauf, M., Dustdar, S., Rosenberg, F.: A survey on context-aware system. International Journal of Ad Hoc and Ubiquitous Computing 1(4), 263-177 (2007)

2. Weber, W., Rabaey, J.M., Aarts, E.: Ambient Intelligence. Springer-Verlag New York, Inc., Heidelberg (2005)

3. Ramos, C., Augusto, J.C., Shapiro, D.: Ambient Intelligence- the Next Step for Artificial Intelligence. IEEE Intelligent Systems 23(2), 15-18 (2008)

4. Raptis, D., Tselios, N., Avouris, N.: Context-based design of mobile applications for museums: a survey of existing practices. In: Proceedings of the 7th International Conference on Human Computer Interaction with Mobile Devices \& Services, Salzburg, Austria, September 19-22 (2005)

5. Abowd, D.A., Atkeson, C.G., Hong, J., Long, S., Pinkerton, M.: Cyberguide: a mobile context-aware tour guide. Wireless Networks 3(5), 421-433 (1996)

6. Oppermann, R., Specht, M.: A context-sensitive nomadic exhibition guide. In: Thomas, P., Gellersen, H.-W. (eds.) HUC 2000. LNCS, vol. 1927, pp. 127-142. Springer, Heidelberg (2000)

7. Bellotti, F., Berta, R., De Gloria, A., Margarone, M.: Guiding Visually Impaired People in the Exhibition. In: Mobile Guide 2006, Turin, Italy (2006)

8. Cheverst, K., Davies, N., Mitchell, K., Smith, P.: Providing tailored Context-aware information to city visitors. In: Brusilovsky, P., Stock, O., Strapparava, C. (eds.) AH 2000. LNCS, vol. 1892, pp. 73-85. Springer, Heidelberg (2000)

9. Blöckner, M., Danti, S., Forrai, J., Broll, G., De Luca, A.: Please touch the exhibits!: using NFC-based interaction for exploring a museum. In: Proceedings of the 11th International Conference on Human-Computer Interaction with Mobile Devices and Services (MobileHCI 2009), Bonn, Germaby, pp. 71-72. ACM, New York (2009)

10. Bruns, E., Brombach, B., Zeidler, T., Bimber, O.: Enabling Mobile Phones To Support Large-Scale Museum Guidance. IEEE Multimedia 14(2), 16-25 (2007)

11. Ghiani, G., Paterno, F., Santoro, C., Spano, L.D.: UbiCicero: A location-aware, multidevice museum guide. Interacting with Computers 21(4), 288-303 (2009)

12. Park, D.-J., Hwang, S.-H., Kim, A.-R., Chang, B.-M.: A Context-Aware Smart Tourist Guide Application for an Old Palace. In: International Conference on Convergence Information Technology, November 21-23, pp. 89-94 (2007)

13. Zimmermann, A., Lorenz, A.: LISTEN: a user-adaptive audio-augmented museum guide. User Modeling and User-Adapted Interaction 18(5), 389-416 (2008)

14. Bay, H., Fasel, B., Van Gool, L.: Interactive museum guide. In: The Seventh International Conference on Ubiquitous Computing UBICOMP, Workshop on Smart Environments and Their Applications to Cultural Heritage (2005)

15. n-Core: A Faster and Easier Way to Create Wireless Sensor Networks (2010), http: / /www.n-core. info (retrieved November 14, 2010) 